\title{
Integrated analysis of lymphocyte infiltration-associated IncRNA for ovarian cancer via TCGA, GTEx and GEO datasets
}

\author{
Meijing Wu ${ }^{\text {Equal first author, } 1}{ }^{\text {, Xiaobin Shang }}{ }^{\text {Equal first author, } 2}{ }^{2}$ Yue Sun ${ }^{1}$, Jing Wu ${ }^{1}$, Guoyan Liu ${ }^{\text {Corresp. } 1}$ \\ ${ }^{1}$ Department of Gynecology and Obstetrics, Tianjin Medical University General Hospital, Tianjin, China \\ 2 Department of Esophageal Cancer, Tianjin Medical University Cancer Institute and Hospital, Tianjin, China \\ Corresponding Author: Guoyan Liu \\ Email address: liuguoyan211@126.com
}

Background: Abnormal expression of long non-coding RNAs (IncRNA) play a significant role in the incidence and progression of high-grade serous ovarian cancer (HGSOC), which is a leading cause of mortality among gynecologic malignant tumor patients. In this study, our aim is to identify IncRNA-associated competing endogenous RNA (ceRNA) axes that could define more reliable prognostic parameters of HGSOC, and to investigate the IncRNAs' potential mechanism of in lymphocyte infiltration. Methods: The RNA-seq and miRNA expression profiles were downloaded from The Cancer Genome Atlas (TCGA) and the Genotype-Tissue Expression (GTEx) database; while for obtaining the differentially expressed IncRNAs (DELs), miRNAs (DEMs), and genes (DEGs), we used edgeR, limma and DESeq2. After validating the RNA, miRNA and gene expressions, using integrated three RNA expression profiles (GSE18520, GSE27651, GSE54388) and miRNA profile (GSE47841) from the Gene Expression Omnibus (GEO) database, we performed Gene Ontology (GO) and Kyoto Encyclopedia of Gene and Genome (KEGG) pathway analyses through ClusterProfiler. The prognostic value of these genes was determined with Kaplan-Meier survival analysis and Cox regression analysis. The ceRNA network was constructed using Cytoscape. The correlation between IncRNAs in ceRNA network and immune infiltrating cells was analyzed by using Tumor IMmune Estimation Resource (TIMER), and gene markers of tumor-infiltrating immune cells were identified using Spearman's correlation after removing the influence of tumor purity. Results: A total of 33 DELs (25 upregulated and 8 downregulated), 134 DEMs (76 upregulated and 58 downregulated), and 1, 612 DEGs (949 upregulated and 663 downregulated) were detected that could be positively correlated with overall survival (OS) of HGSOC. With the 1, 612 analyzed genes, we constructed a ceRNA network, which indicated a pre-dominant involvement of the immune-related pathways. Furthermore, our data revealed that LINC00665 influenced the infiltration level of macrophages and dendritic cells (DCs). On the other hand, FTX and LINC00665, which may play their possible roles through the ceRNA axis, demonstrated a Peer) reviewing PDF | (2019:12:44443:1:0:REVIEW 3 Mar 2020) 
potential to inhibit Tregs and prevent T-cell exhaustion. Conclusion: We defined several prognostic biomarkers for the incidence and progression of HGSOC and constructed a network for ceRNA axes; among which three were indicated to have a positive correlation with lymphocyte infiltration, namely; FTX-hsa-miR-150-5p-STK11, LINC00665-hsamiR449b-5p-VAV3 and LINC00665-hsa-miR449b-5p-RRAGD. 


\section{Integrated analysis of lymphocyte infiltration-}

2 associated IncRNA for ovarian cancer via TCGA, GTEx

3 and GEO datasets

${ }^{1}$ Department of Gynecology and Obstetrics, Tianjin Medical University General Hospital,

9 Tianjin, 300052, China; ${ }^{2}$ Department of Esophageal Cancer, Tianjin Medical University Cancer

10 Institute and Hospital, Tianjin, 300060, China.

11 a Meijing $\mathrm{Wu}$ and Xiaobin Shang contributed equally to this work.

12

Corresponding Author:

Dr. Guoyan Liu.

Department of Gynecology and Obstetrics, Tianjin Medical University General Hospital,

16 Tianjin, 300052, China.

17 E-mail: liuguoyan211@126.com. 
19

20

21

22

23

24

25

26

27

28

29

30

31

32

33

34

35

36

37

38

39

40

41

42

43

44

45

46

47

48

49

50

51

52

53

54

55

56

57

58

\section{Abstract}

Background: Abnormal expression of long non-coding RNAs (lncRNA) play a significant role in the incidence and progression of high-grade serous ovarian cancer (HGSOC), which is a leading cause of mortality among gynecologic malignant tumor patients. In this study, our aim is to identify lncRNA-associated competing endogenous RNA (ceRNA) axes that could define more reliable prognostic parameters of HGSOC, and to investigate the lncRNAs' potential mechanism of in lymphocyte infiltration.

Methods: The RNA-seq and miRNA expression profiles were downloaded from The Cancer Genome Atlas (TCGA) and the Genotype-Tissue Expression (GTEx) database; while for obtaining the differentially expressed lncRNAs (DELs), miRNAs (DEMs), and genes (DEGs), we used edgeR, limma and DESeq2. After validating the RNA, miRNA and gene expressions, using integrated three RNA expression profiles (GSE18520, GSE27651, GSE54388) and miRNA profile (GSE47841) from the Gene Expression Omnibus (GEO) database, we performed Gene Ontology (GO) and Kyoto Encyclopedia of Gene and Genome (KEGG) pathway analyses through ClusterProfiler. The prognostic value of these genes was determined with Kaplan-Meier survival analysis and Cox regression analysis. The ceRNA network was constructed using Cytoscape. The correlation between lncRNAs in ceRNA network and immune infiltrating cells was analyzed by using Tumor IMmune Estimation Resource (TIMER), and gene markers of tumor-infiltrating immune cells were identified using Spearman's correlation after removing the influence of tumor purity.

Results: A total of 33 DELs (25 upregulated and 8 downregulated), 134 DEMs (76 upregulated and 58 downregulated), and 1, 612 DEGs (949 upregulated and 663 downregulated) were detected that could be positively correlated with overall survival (OS) of HGSOC. With the 1, 612 analyzed genes, we constructed a ceRNA network, which indicated a pre-dominant involvement of the immune-related pathways. Furthermore, our data revealed that LINC00665 influenced the infiltration level of macrophages and dendritic cells (DCs). On the other hand, FTX and LINC00665, which may play their possible roles through the ceRNA axis, demonstrated a potential to inhibit Tregs and prevent T-cell exhaustion.

Conclusion: We defined several prognostic biomarkers for the incidence and progression of HGSOC and constructed a network for ceRNA axes; among which three were indicated to have a positive correlation with lymphocyte infiltration, namely; FTX-hsa-miR-150-5p-STK11, LINC00665-hsa-miR449b-5p-VAV3 and LINC00665-hsa-miR449b-5p-RRAGD.

\section{Introduction}

In USA, among the gynecologic malignant tumors, the highest mortality in 2019 was been reported to be ovarian cancer, related with an annual incidence of 22,530 and a mortality of 13,980. This is significantly higher than cervical or endometrial cancers (Siegel et al., 2019). Most deaths (70\%) were presented with an advanced-stage, high-grade serous ovarian cancer (HGSOC). The standard treatment encompasses surgery combined with platinum-based chemotherapy. However, most patients died of platinum-resistance followed by cancer 
59

60

61

62

63

64

65

66

67

68

69

70

71

72

73

74

75

76

77

78

79

80

81

82

83

84

85

86

87

88

89

90

91

92

93

94

95

96

97

98

recurrence (Liu et al., 2015). Therefore, it is of great significance to study the underlying molecular mechanisms of the development of ovarian cancer, which can help us identify more molecular markers for monitoring recurrence, as well as exploring more effective ways to evade drug resistance, and control progression or metastasis.

Accumulating evidence has demonstrated that abnormal expression of long non-coding RNA (lncRNA), which is more specific to tissues and has more quantities in comparison with proteincoding genes (Cabili et al., 2011), is involved in the carcinogenesis and progression of ovarian cancer (Martini et al., 2017; Wu et al., 2017). The lncRNAs can sponge a miRNA through the miRNA response elements (MREs) thereby inhibiting the translation of mRNA, which is propagated as the competing endogenous RNA (ceRNA) hypothesis (Thomson \& Dinger, 2016). This regulatory mechanism also plays an important role in the development of ovarian cancer. For instance, MALAT1- miR-506- iASPP axis was found to regulate the ovarian cancer growth and DNA synthesis (Lei et al., 2017). NEAT1 expression knockdown in platinum resistant $\left(\right.$ PTX $^{r}$ ) ovarian cancers can improve PTX sensitivity, which was mediated by miR-194/ZEB1 axis (An et al., 2017). The overexpression of IncRNA HOTAIR and signaling protein MAPK1 can be reversed by upregulating miR-1, miR-214-3p, or miR-330-5p, indicating a partially attribution to the ceRNA regulatory network (Yiwei et al., 2015). However, to date, the studies performed to investigate competing endogenous RNA mechanisms of lncRNAs for HGSOC are rare.

The goal of this investigation was to identify the prognostic value of the lncRNA-miRNAmRNA interaction axes for elucidating the development of HGSOC, as well as investigating the potential mechanisms of lncRNAs in lymphocyte infiltration. We have identified novel prognostic biomarkers and therapeutic targets for the HGSOC treatment.

(1)

\section{Materials \& Methods}

\section{Data collection and preprocessing}

The RNA-seq and miRNA expression profiles of ovarian cancer were downloaded from The Cancer Genome Atlas (TCGA), while the RNA-seq datasets of normal ovarian tissue were searched from the Genotype-Tissue Expression (GTEx) (Carithers et al., 2015). The RNA-seq datasets of counts value from TCGA and GTEx were normalized and processed with 9 TCGAbiolinks package (Mounir et al., 2019) (Table S1). It contains RNA expression profile of 363 high grade serous ovarian cancer and 108 normal tissues. The miRNA datasets from TCGA 1 contain microRNA expression profiles of 479 HGSOC and 8 normal tissues (Table S2). On the 2 basis of the information recorded in the HUGO Gene Nomenclature Committee (HGNC; 3 http://www.genenames.org/) (Eyre et al., 2006), the lncRNAs and protein-coding genes in RNA 4 expression profiles were annotated. Figure S1 illustrates the workflow for bioinformatics based analysis.

\section{Differential RNA expression analysis}

7 The differentially expressed protein-coding genes/lncRNAs (DEGs/DELs) from HGSOC and 8 normal ovarian tissues were selected using the edgeR (Version 3.22.5; 
http://www.bioconductor.org/pack-ages/release/bioc/html/edgeR.html), limma (Version 3.30.0; http://www.bioconductor.org/packages/release/bioc/html/limma.html) and DESeq2 (Version 1.20.0; http://www.bioconductor.org/packages/release/bioc/html/DESeq2.html) package of $\mathrm{R}$ software (Nikolayeva \& Robinson, 2014; Carvalho et al., 2007; Anders \& Huber, 2010). Subsequently, we combined the differentially expressed genes, acquired from the three packages, to get the convergence gene set. On the other hand, differentially expressed miRNAs (DEMs) were identified using the limma package. A false discovery rate (FDR) $<0.05$ and $\mid \log F C$ (fold change) $\mid>1$ were set as the criteria value for DEGs, DEMs and DELs. The expression intensity and direction of DEGs, DELs, and DEMs were represented by heatmaps using the pheatmap R package (Version: 1.0.12; https://cran.r-project.org/web/packages/pheatmap) based on Euclidean distance.

\section{Validation of DEL, DEM, and DEG expressions}

We chose four ovarian cancer datasets from the Gene Expression Omnibus (GEO) database (http://www.ncbi.nlm.nih.gov/geo/). Three of them (GSE18520: 53 vs 10, GSE27651: 22 vs 6, GSE54388: 16 vs 6) were based on the GPL570 platform (Affymetrix Human Genome U133 Plus 2.0 Array) while another one (GSE47841: 12 vs 9) was from GPL14613 platform (Affymetrix Multispecies miRNA-2 Array). In order to improve the number of samples (91 HGSOC samples vs 22 normal samples), we integrated all samples of three datasets (GSE18520, GSE27651, GSE54388), and then removed the batch effect so as to avoid generating less reliable results using sva package (Leek et al., 2012) ( Table S3). Principal Component Analysis (PCA) was performed on the gene expression profiles obtained from GEO, to reduce the dimensionality and evaluate the independence of normal and tumor samples. The expressions of DELs, DEMs and DEGs were validated in microarray datasets from GEO that detected expression profiles containing mRNA and lncRNA (GSE18520, GSE27651, GSE54388) as well as miRNA (GSE47841) between the HGSOC and normal tissues. The difference of expression was tested by t-test. A value of $p<0.05$ was considered statistically significant.

\section{Function enrichment analysis}

Biological process of Gene Ontology (GO) and Kyoto Encyclopedia of Genes and Genomes (KEGG) analysis of DEGs was conducted by using the clusterProfiler R package (Version 6.8; http://www.bioconductor.org/packages/release/bioc/html/clusterProfiler.html) (Yu et al., 2012) to predict their underlying functions. A $p<0.05$ was considered to be statistically significant.

\section{Survival analysis}

The expressions of DEG/DEL/DEM were extracted and merged with the survival information in each sample (Table S4). Univariate Cox regression analysis was implemented to perform prognostic analysis using the R survival package (Version 2.43.3; https://cran.rproject.org/package=survival). The survival result was expressed as Kaplan-Meier (K-M) curve, with higher expression of a specific gene versus lower expression of this gene in a patient, the cutoff value determined by survminer (Version 0.4.3; https://cran.rproject.org/package $=$ survminer). Again a $p<0.05$ was considered as related to prognostic significance. 


\section{IncRNA-miRNA-mRNA ceRNA regulatory network construction}

140 The starBase database (http://starbase.sysu.edu.cn/) (Li et al., 2014) was used to study the 141 IncRNA-miRNA interactions. The target genes of miRNAs in the DELs-DEMs interaction 142 network were predicted using the miRTarBase database. The negative interaction pairs between 143 DEMs and DEGs/DELs were integrated to construct the DELs-DEMs-DEGs ceRNA network 144 using the Cytoscape software (Version 3.6.1; www.cytoscape.org/) (Kohl et al., 2011).

145 Furthermore, we analyzed functional enrichment of the genes in the ceRNA network with 146 clusterProfiler R package.

147 Immune infiltrates analysis

148 We analyzed the correlation between lncRNAs in ceRNA network and the abundance of immune 149 cell infiltrates; including B cells, $\mathrm{CD}^{4+} \mathrm{T}$ cells, $\mathrm{CD}^{8+} \mathrm{T}$ cells, neutrophils, macrophages, and 150 dendritic cells using Tumor IMmune Estimation Resource (TIMER)

151

152

153

154

155

156

157

158

159

160

161

162

163

164

165

166

167

168

169

170

171

172

173

174

175

176

177

178 (https://cistrome.shinyapps.io/timer/) (Li et al., 2017). And the tumor purity was calculated using the estimate R package (https://r-forge.r-project.org/projects/estimate/), In addition, correlations between the lncRNAs and marker genes of tumor-infiltrating lymphocytes were explored using Spearman's correlation. The gene markers of tumor-infiltrating lymphocytes included markers of $\mathrm{CD}^{8+} \mathrm{T}$ cells, B cells, general T cells, tumor-associated macrophages (TAMs), M1 macrophages, M2 macrophages, monocytes, neutrophils, natural killer (NK) cells, dendritic cells (DC), T helper 1 (Th1) cells, T helper 2 (Th2) cells, T helper 17 (Th17) cells, follicular helper T (Tfh) cells, regulatory $\mathrm{T}$ cell (Tregs) and exhausted $\mathrm{T}$ cells, which were referenced in prior studies (Sousa \& Maatta, 2016). The gene expression level is displayed with $\log 2$ transformation.

\section{Results}

\section{Differential expression analysis}

A total of 629 DELs (343 downregulated and 286 upregulated), 7, 514 DEGs (3, 319 downregulated and 4, 195 upregulated) and 336 DEMs (165 downregulated and 171 upregulated) were identified between HGSOC and normal ovarian tissues based on the threshold of FDR $<0.05$ and $|\operatorname{logFC}|>1$. The expression changes of DELs (Figure. S2A), DEMs (Figure S2B), and DEGs (Figure S2C) were displayed by heatmap analysis.

\section{Validation of DEL, DEM, and DEG expressions}

To validate the expressions of detected differently expressed genes above, we utilized datasets from GEO. The results of PCA indicated that normal samples vs. tumor samples in the merged datasets had a significant difference (Figure S3). After combining gene expressions in GEO, 111 DELs (77 downregulated and 34 upregulated), 2, 620 DEGs (1, 535 downregulated and 1, 085 upregulated) while 186 DEMs (101 downregulated and 85 upregulated) were identified for subsequent analysis.

\section{Functional annotation for DEGs}

GO and KEGG pathway analysis were performed to explore the potential biological functions of DEGs. The results, through KEGG pathway enrichment analysis, indicated that upregulated genes were mainly involved in cell cycle, oxidative phosphorylation, homologous 
179 recombination, cytokine-cytokine receptor interaction and IL-17 signaling pathway, while

180 downregulated genes mainly enriched in cGMP-PKG signaling pathway, complement and 181 coagulation cascades, TGF- $\beta$ signaling pathway and Ras signaling pathway, as shown in Figure

182 1A and Table S5. Furthermore, the GO biological process terms were performed. In line with the 183 KEGG enrichment results, regulation of cell cycle phase transition and positive regulation of cell 184 cycle were obtained for the upregulated genes, while transmembrane receptor protein

185

186

187

188

189

190

191

192

193

194

195

196

197

198

199

200

201

202

203

204

205

206

207

208

209

210

211

212

213

214

215

216

217

218

serine/threonine kinase signaling pathway and regulation of membrane lipid distribution was enriched in the downregulated genes (Figure 1B and Table S6).

\section{Prognosis prediction for DELs, DEMs, and DEGs}

We further performed the survival analysis for DELs, DEMs, and DEGs base on TCGA data and clinical information to excavate the prognostic genes. The results showed that 33 DELs (25 upregulated, 8 downregulated), 134 DEMs (76 upregulated, 58 downregulated), and 1612 DEGs (949 upregulated, 663 downregulated) were significantly associated with overall survival (OS) $(p<0.05)$ (Table S7).

\section{ceRNA network construction}

We then constructed ceRNA networks to explore the interactions between DELs, DEMs and DEGs. The 33 OS-related DELs were mapped into the starBase database to predict their target microRNAs. Comparing with the OS-related DEMs, lncRNA/miRNA interacted networks were constructed. Subsequently, the target genes of selected DEMs were searched using miRTarBase database for identified DEMs-DEGs interaction. After taking the change of expression and prognosis information into consideration, several DELs-DEMs-DEGs interactions including 4 DELs (3 upregulated, 1 downregulated), 8 DEMs (3 upregulated, 5 downregulated) and 44 DEGs (15 upregulated, 29 downregulated) were predicted and resultantly a DELs-DEMs-DEGs ceRNA network was established, as shown in Figure 2.

Function enrichment analysis showed the genes in the ceRNA network participate in several KEGG pathways; including T cell receptor signaling pathway, MicroRNAs in cancer progression pathway, FoxO signaling pathway, Autophagy-animal and mTOR signaling pathways (Table S8). The DEL-DEM-DEG interactions associated with signaling pathways are shown in Figure 3, which involved 3 DELs (2 upregulated and 1 downregulated), 4 DEMs (2 upregulated and 2 downregulated), and 7 DEGs (4 upregulated and 3 downregulated).

Further K-M curves of genes in the ceRNA network were plotted. The relationships between their respective expressions and the prognostic values were in accordance with our hypothesis. The results showed that patients with a high expression of DELs (LINC00858 and FTX) and DEGs (PAK4, ZEB1, STK11 and SETD7) indicated a poor survival prognosis, meanwhile the high expressions of DEMs (has-miR-324-5p, has-miR-150-5p and has-miR-342-3p) in the ceRNA axes presented and good survival prognosis. Similarly, the high expressions of LINC00665 and the related protein coding genes (VAV3, E2F3, RRAGD) played their roles as suppressor genes, while the high expressions of has-miR-449-5p acted as an oncogene (Figure. 4 and Figure S4).

IncRNA expression is correlated with lymphocyte infiltration level in HGSOC 
219 Since the functional analysis indicated that the mRNAs in ceRNA network enriched in immune-

220

221

222

223

224

225

226

227

228

229

230

231

232

233

234

235

236

237

238

239

240

241

242

243

244

245

246

247

248

249

250

251

252

253

254

255

256

257

258

related pathways, such as T-cell receptor signaling pathway, Autophagy - animal, and mTOR signaling pathway, whether the lnRNAs played potential roles in lymphocyte infiltration?

Tumor-Infiltrating Lymphocyte Grade has been proven to be an independent predictor of sentinel lymph node status, as well as survival in cancers (Ohtani, 2007; Azimi et al., 2012). However, genomic approaches that are commonly used for the analysis of lymphocyte infiltration in clinical tumor samples can be severely influenced by tumor purity (Yoshihara et al., 2013). We put the expression profiles into TIMER website after eliminating the influence of tumor purity. The results showed that expression levels of three lncRNAs all have significantly positive correlations with infiltrating levels of tumor purity (Figure S5, first column). FTX exhibited significant negative correlation with infiltrating levels of $\mathrm{CD}^{8+} \mathrm{T}$ cells $(\mathrm{r}=-0.13, \mathrm{P}=0.012)$, neutrophils $(\mathrm{r}=-0.12, \mathrm{P}=0.018)$, and macrophages $(\mathrm{r}=-0.17, \mathrm{P}=0.00091)$ as shown in Figure 5A. LINC00855 also has negative correlations with neutrophils $(\mathrm{r}=-0.12, \mathrm{P}=0.021)$ and $\mathrm{DCs}(\mathrm{r}$ $=-0.11, \mathrm{P}=0.043$ ) (Figure. 5B). On the other hand, LINC00665 demonstrated significant positive correlation with infiltrating levels of $\mathrm{CD}^{4+} \mathrm{T}$ cells $(\mathrm{r}=0.11, \mathrm{P}=0.044)$, negative correlations with $\mathrm{CD}^{8+} \mathrm{T}$ cells $(\mathrm{r}=-0.16, \mathrm{P}=0.0026)$, neutrophils $\left(\mathrm{r}=-0.27, \mathrm{P}=1.8^{\mathrm{e}-07}\right)$, macrophages $(r=-0.11, P=0.029)$ and DCs $(r=-0.26, P=3.7 e-07)$ (Figure. 5C). These findings suggest that three lncRNAs, LINC00855, FTX and LINC00665, play a specific role in lymphocyte infiltration in HGSOC, especially LINC00665.

\section{Correlation analysis between IncRNA expression and immune marker sets}

We further investigated the correlations between lncRNAs and marker sets of different immune cells, including $\mathrm{CD}^{8+} \mathrm{T}$ cells, $\mathrm{T}$ cells (general), B cells, monocytes, TAMs, M1 and M2 macrophages, neutrophils, NK cells, and DCs. Different type of T cells were also analyzed, which include Th1 cells, Th2 cells, Tfh cells, Th17 cells, Tregs, as well as exhausted T cells (Table 1 and Table S9).

After adjusting correlation by purity, the results revealed that most immune markers of various immune cells and T cells are significantly correlated with the FTX and LINC00665 expression level. However, only 22 gene markers were found to be correlated with the expression level of LINC00885.

It is also found that the expression levels of most markers of monocytes, TAMs, M2 macrophages have strong correlations with LINC00665 expression.

As is shown in Figure 5, high LINC00665 expression correlates with low infiltration level of DCs. This result can be explained by the following findings that DC markers such as HLADPB1, HLA-DQB1, HLA-DRA, HLA-DPA1, CD1C (BDCA-1) and ITGAX (CD11c) show significant negative correlations with LINC00665 expression. We can further conclude that there is a significant negative correlation between LINC00665 and DC infiltration. This finding is rather interesting because tumor metastasis can be aggravated by $\mathrm{DC}$ reducing $\mathrm{CD}^{8+} \mathrm{T}$ cell cytotoxicity and increasing Treg cells (Sawant et al., 2012).

It is also found that there are significant correlations between FTX, LINC00665 and marker genes of Treg and T cell exhaustion, such as FOXP3, TGFB1 (TGF $\beta$ ), IL10, TBX21 (T-bet), 
259

260

261

262

263

264

265

266

267

268

269

270

271

272

273

274

275

276

277

278

279

280

281

282

283

284

285

286

287

288

289

290

291

292

293

294

295

296

297

PDCD1 (PD-1), CTLA4 and HAVCR2 (TIM-3). FOXP3 is a critical marker of Treg cells which suppress cytotoxic T cells attacking tumor cells (Facciabene et al., 2012). We found that FTX and LINC00665 expressions have strong negative correlations with TIM-3, which is known as a critical factor that regulates $\mathrm{T}$ cell exhaustion. This finding suggests that these two lncRNA expressions are of great importance in TIM-3 mediating T cell exhaustion (Table 1). Accordingly, it is further confirmed that FTX and LINC00665 are specifically correlated with immune infiltrating cells, suggesting that these two lncRNAs play vital roles in inhibiting immune escape in the ovarian microenvironment.

\section{Discussion}

It is increasingly clear that abnormal lncRNA expression might contribute to tumorigenesis and progression of high-grade serous ovarian cancer (HGSOC). The ceRNA hypothesis, in which lncRNAs play their role at the post-transcriptional level, has been considered as a novel measure of gene regulation. However, comprehensive analyses of differentially expressed profiles of lncRNAs, miRNAs and mRNA, as well as their interactions through integrating TCGA, GTEx and GEO datasets have not yet been reported.

In the present study, we obtained complete datasets of IncRNA, microRNA and mRNA through differential gene expression analysis after analyzing datasets from TCGA and GTEx and integrated validation datasets from GEO. And then these genes were submitted for survival analysis for identifying OS-associated DELs, DEMs and DEGs. We further developed a prognostic ceRNA network with the detected OS-associated genes, which contain 33 DELs (25 upregulated, 8 downregulated), 134 DEMs (76 upregulated, 58 downregulated) and 1, 612 DEGs (949 upregulated, 663 downregulated).

The ceRNA network was constructed by using four IncRNAs, LINC01224, FTX, LINC00855 and LINC00665. Considering that mRNAs are the implementers of molecular function, KEGG analysis were performed to annotate the functions of DEGs influenced by the four IncRNAs in the ceRNA network. Three lncRNAs, LINC00665, LINC00855 and FTX, were left with their target genes involved in several pathways, one of which was miRNA in cancer pathway, indicating that they may act partly in a ceRNA network to exert their functional role.

To get a better understanding of the molecular functions of these three OS-associated lncRNAs, we reviewed the published researches of the same lncRNAs. LINC00665 is upregulated in HGSOC, it is also overexpressed in hepatocellular carcinoma (HCC) and lung cancer patients, but act as an oncogene with poor prognosis (Wen et al., 2018), which is contrary to our univariate Cox regression analysis (HR:0.89, 95\%CI:0.73-1.1). In lung adenocarcinoma (LUAD) tissues, it played its roles through linc00665-miR98-AKR1B10 axis (Cong et al., 2019). LINC00858 expression was observed to be up-regulated in colorectal cancer (CRC), osteosarcoma and non-small cell lung cancer (NSCLC) tissues, and associated with poor prognosis, which is in line with our hypothesis, and it exerted its functional effect through the miR-22-3p/YWHAZ axis in CRC (Sha et al., 2019). thus promoting tumorigenesis through 
298 LINC00858/miR-139/CDK14 axis in osteosarcoma ( $G$ u et al., 2018), as a ceRNA for miR-422a

299 to facilitate cell proliferation in NSCLC (Zhu et al., 2017).

300 The long non-coding RNA FTX is an X-inactive-specific transcript (XIST) regulator transcribed 301 from the $\mathrm{X}$ chromosome inactivation center. Similar to our results, a significant decrease of FTX 302 in HCC tissues was observed, and patients with higher FTX expression exhibited longer survival, 303 it acted as a tumor suppressor through binding with miR-374a and MCM2 (Liu et al., 2016).

304 While in another research, Li et. al reported that FTX was upregulated in HCC, acting as a 305 promoter of tumor progression ( $L i$ et al., 2018). And it was also upregulated in CRC, gliomas, 306 renal cell carcinoma (RCC) tissue and indicated poor prognosis of patients with CRC (Yang et 307 al., 2018) and RCC (He et al., 2017), it bound miR-215 and vimentin in CRC, negatively 308 regulating miR-342-3p in gliomas to promote proliferation and invasion (Zhang et al., 2017). 309 miR-342-3p was also predicted to interact with FTX in our result.

310 These results indicate that LINC00665, LINC00855 and FTX can perform their function through 311 miRNA competition as a part of ceRNA axes. But more experiments are needed to validate the 312 roles of these ceRNA axes in HGSOC.

313 The tumorigenesis and progression not only ascribe to gene variation, but also have strong 314 connection with tumor microenvironment. The tumor-infiltrating lymphocytes (TILs), as 315 components of ovarian cancer tumor microenvironment, have already been shown to have 316 correlation with OS. A previous study divided HGSOC into four molecular subtypes namely, 317 "immunoreactive," "differentiated," "proliferative," and "mesenchymal" on the basis of gene 318 expressions obtained from TCGA. And it is found that the prognosis of immunoreactive subtype 319 is better than the other subtypes (Konecny et al., 2014), indicating the immune factors are of vital 320 importance in the development of HGSOC.

321 Interestingly, the KEGG analysis showed that DELs and their target DEGs were associated with 322 immune-related pathway, such as T cell receptor signaling pathway, Autophagy and mTOR 323 signaling pathway, suggesting the correlations between the DELs and the activation of the 324 immune system.

325 Taking advantage of the website TIMER and co-expression analysis, we found that there were 326 negative correlations between LINC00665 expression level and infiltration level of DCs and 327 macrophages. Moreover, the relationships between LINC00665 expression and the gene markers 328 of immune cells supported the result of lymphocyte infiltration. First, gene markers of M1 329 macrophages such as NOS2 (INOS), IRF5 and PTGS2 (COX2) were weakly correlated with 330 LINC00665 expression, whereas markers of M2 macrophage such as CD163, MS4A4A and 331 VSIG4 showed moderate and strong correlations. These results revealed that LINC00665 may 332 regulate the tumor-associated macrophage (TAM) polarization.

333 Our results also demonstrated that FTX and LINC00665 had the potential to inhibit Tregs and 334 prevent T cell exhaustion. The decreased FTX and increased LINC00665 expression negatively 335 correlated with the expression of Treg and T cell exhaustion markers (FOXP3, TGFB1 (TGF $\beta$ ), 336 IL10, TBX21 (T-bet), PDCD1 (PD-1), CTLA4 and HAVCR2 (TIM-3)). 
337 FTX and LINC00665 also regulated several marker genes of T helper cells (Th1, Th2, Th17 and

$338 \mathrm{Tfh})$. These correlations could reveal a potential mechanism where they regulate $\mathrm{T}$ cell functions

339 in HGSOC. Together, these findings suggested that FTX and LINC00665, especially

340 LINC00665, play important roles in regulation of immune infiltrating cells in HGSOC.

341 Our result defined several ceRNA axes that may influence the tumor immune microenvironment.

342 FTX-hsa-miR-150-5p-STK11 was associated with mTOR signaling pathway and autophagy.

343 STK11 (LKB1) inhibited mTOR signaling. STK11 silencing inhibited autophagy induction and

344 tumor inhibition (Chung et al., 2017). Akt/mTOR signaling pathway is a well-known pathway

345 which negatively regulates autophagy (Hanahan \& Weinberg, 2000). mTOR is an evolutionarily

346 conserved serine-threonine kinase, which has emerged as a central regulator of T-cell lineage

347 specification (Delgoffe et al., 2011). In particular, constitutively active mTOR and Akt caused

348 abrogation of Treg differentiation (Haxhinasto et al., 2008).

349 LINC00665-hsa-miR449b-5p-RRAGD was associated with mTOR signaling pathway and

350 autophagy as well. RRAGD promoted mTORC1 activity and tumor growth (Di Malta et al.,

351 2017), influencing the T-cell similarly. LINC00665-hsa-miR449b-5p-VAV3 was relative with T

352 cell receptor signaling pathway. Researchers observed signal-induced tyrosine phosphorylation

353 of VAV1 and VAV3, which are cytosolic signaling scaffolds and guanine nucleotide exchange

354 factors that can play context-specific roles in immune receptor pathways (Bustelo, 2014).

355

356

\section{Conclusions}

357 In summary, we defined two immune-associated lncRNAs, FTX and LINC00665, which can act

358 as prognostic biomarkers in HGSOC, and constructed three ceRNA axes: FTX-hsa-miR-150-5p-

359 STK11, LINC00665-hsa-miR449b-5p-VAV3 and LINC00665-hsa-miR449b-5p-RRAGD. 
361

362

363

364

365

366

367

368

369

370

371

372

373

374

375

376

377

378

379

380

381

382

383

384

385

386

387

388

389

390

391

392

393

394

395

396

397

398

399

\section{References}

Siegel RL, Miller KD, and Jemal A. 2019. Cancer statistics, 2019. CA Cancer J Clin 69:7-34. DOI 10.3322/caac.21551

Liu G, Xue F, and Zhang W. 2015. miR-506: a regulator of chemo-sensitivity through suppression of the RAD51-homologous recombination axis. Chin J Cancer 34:485-487. DOI 10.1186/s40880-015-0049-z

Cabili MN, Trapnell C, Goff L, Koziol M, Tazon-Vega B, Regev A, and Rinn JL. 2011. Integrative annotation of human large intergenic noncoding RNAs reveals global properties and specific subclasses. Genes Dev 25:1915-1927. DOI 10.1101/gad.17446611

Martini P, Paracchini L, Caratti G, Mello-Grand M, Fruscio R, Beltrame L, Calura E, Sales G, Ravaggi A, Bignotti E, Odicino FE, Sartori E, Perego P, Katsaros D, Craparotta I, Chiorino G, Cagnin S, Mannarino L, Ceppi L, Mangioni C, Ghimenti C, D'Incalci M, Marchini S, and Romualdi C. 2017. IncRNAs as Novel Indicators of Patients' Prognosis in Stage I Epithelial Ovarian Cancer: A Retrospective and Multicentric Study. Clin Cancer Res 23:2356-2366. DOI 10.1158/1078-0432.CCR-16$\underline{1402}$

Wu DD, Chen X, Sun KX, Wang LL, Chen S, and Zhao Y. 2017. Role of the lncRNA ABHD11-AS1 in the tumorigenesis and progression of epithelial ovarian cancer through targeted regulation of RhoC. Mol Cancer 16:138. DOI 10.1186/s12943-017-0709-5

Thomson DW, and Dinger ME. 2016. Endogenous microRNA sponges: evidence and controversy. Nat Rev Genet 17:272-283. DOI 10.1038/nrg.2016.20

Lei R, Xue M, Zhang L, and Lin Z. 2017. Long noncoding RNA MALAT1-regulated microRNA 506 modulates ovarian cancer growth by targeting iASPP. Onco Targets Ther 10:35-46. DOI 10.2147/ott.s112686

An J, Lv W, and Zhang Y. 2017. LncRNA NEAT1 contributes to paclitaxel resistance of ovarian cancer cells by regulating ZEB1 expression via miR-194. Onco Targets Ther 10:5377-5390. DOI 10.2147/OTT.S147586

Yiwei T, Hua H, Hui G, Mao M, and Xiang L. 2015. HOTAIR Interacting with MAPK1 Regulates Ovarian Cancer skov3 Cell Proliferation, Migration, and Invasion. Med Sci Monit 21:1856-1863. DOI 10.12659/MSM.893528

Carithers LJ, Ardlie K, Barcus M, Branton PA, Britton A, Buia SA, Compton CC, DeLuca DS, Peter-Demchok J, Gelfand ET, Guan P, Korzeniewski GE, Lockhart NC, Rabiner CA, Rao AK, Robinson KL, Roche NV, Sawyer SJ, Segre AV, Shive CE, Smith AM, Sobin LH, Undale AH, Valentino KM, Vaught J, Young TR, Moore HM, and Consortium GT. 2015. A Novel Approach to High-Quality Postmortem Tissue Procurement: The GTEx Project. Biopreserv Biobank 13:311-319. DOI 10.1089/bio.2015.0032

Mounir M, Lucchetta M, Silva TC, Olsen C, Bontempi G, Chen X, Noushmehr H, Colaprico A, and Papaleo E. 2019. New functionalities in the TCGAbiolinks package 
400

401

402

403

404

405

406

407

408

409

410

411

412

413

414

415

416

417

418

419

420

421

422

423

424

425

426

427

428

429

430

431

432

433

434

435

436

437

438

439

for the study and integration of cancer data from GDC and GTEx. PLoS Comput Biol 15:e1006701. DOI 10.1371/journal.pcbi.1006701

Eyre TA, Ducluzeau F, Sneddon TP, Povey S, Bruford EA, and Lush MJ. 2006. The HUGO Gene Nomenclature Database, 2006 updates. Nucleic Acids Res 34:D319-321. DOI 10.1093/nar/gkj147

Nikolayeva O, and Robinson MD. 2014. edgeR for differential RNA-seq and ChIP-seq analysis: an application to stem cell biology. Methods Mol Biol 1150:45-79. DOI 10.1007/978-1-4939-0512-6 3

Carvalho B, Bengtsson H, Speed TP, and Irizarry RA. 2007. Exploration, normalization, and genotype calls of high-density oligonucleotide SNP array data. Biostatistics 8:485-499. DOI 10.1093/biostatistics/kx1042

Anders S, and Huber W. 2010. Differential expression analysis for sequence count data. Genome Biol 11:R106. DOI 10.1186/gb-2010-11-10-r106

Leek JT, Johnson WE, Parker HS, Jaffe AE, and Storey JD. 2012. The sva package for removing batch effects and other unwanted variation in high-throughput experiments. Bioinformatics 28:882-883. DOI 10.1093/bioinformatics/bts034

Yu G, Wang LG, Han Y, and He QY. 2012. clusterProfiler: an R package for comparing biological themes among gene clusters. OMICS 16:284-287. DOI $\underline{10.1089 / \text { omi.2011.0118 }}$

Li JH, Liu S, Zhou H, Qu LH, and Yang JH. 2014. starBase v2.0: decoding miRNA-ceRNA, miRNA-ncRNA and protein-RNA interaction networks from large-scale CLIP-Seq data. Nucleic Acids Res 42:D92-97. DOI 10.1093/nar/gkt1248

Kohl M, Wiese S, and Warscheid B. 2011. Cytoscape: software for visualization and analysis of biological networks. Methods Mol Biol 696:291-303. DOI 10.1007/978-1-60761-987118

Li T, Fan J, Wang B, Traugh N, Chen Q, Liu JS, Li B, and Liu XS. 2017. TIMER: A Web Server for Comprehensive Analysis of Tumor-Infiltrating Immune Cells. Cancer Res 77:e108-e110. DOI 10.1158/0008-5472.CAN-17-0307

Sousa S, and Maatta J. 2016. The role of tumour-associated macrophages in bone metastasis. $J$ Bone Oncol 5:135-138. DOI 10.1016/j.jbo.2016.03.004

Ohtani H. 2007. Focus on TILs: prognostic significance of tumor infiltrating lymphocytes in human colorectal cancer. Cancer Immun 7:4.

Azimi F, Scolyer RA, Rumcheva P, Moncrieff M, Murali R, McCarthy SW, Saw RP, and Thompson JF. 2012. Tumor-Infiltrating Lymphocyte Grade Is an Independent Predictor of Sentinel Lymph Node Status and Survival in Patients With Cutaneous Melanoma. Journal Of Clinical Oncology 30:2678-2683. DOI 10.1200/Jco.2011.37.8539

Yoshihara K, Shahmoradgoli M, Martinez E, Vegesna R, Kim H, Torres-Garcia W, Trevino V, Shen H, Laird PW, Levine DA, Carter SL, Getz G, Stemke-Hale K, Mills GB, and Verhaak RG. 2013. Inferring tumour purity and stromal and immune cell admixture from expression data. Nat Commun 4:2612. DOI 10.1038/ncomms3612 
440

441

442

443

444

445

446

447

448

449

450

451

452

453

454

455

456

457

458

459

460

461

462

463

464

465

466

467

468

469

470

471

472

473

474

475

476

477

478
Sawant A, Hensel JA, Chanda D, Harris BA, Siegal GP, Maheshwari A, and Ponnazhagan S. 2012. Depletion of plasmacytoid dendritic cells inhibits tumor growth and prevents bone metastasis of breast cancer cells. J Immunol 189:4258-4265. DOI 10.4049/jimmunol.1101855

Facciabene A, Motz GT, and Coukos G. 2012. T-regulatory cells: key players in tumor immune escape and angiogenesis. Cancer Res 72:2162-2171. DOI 10.1158/00085472.CAN-11-3687

Wen DY, Lin P, Pang YY, Chen G, He Y, Dang YW, and Yang H. 2018. Expression of the Long Intergenic Non-Protein Coding RNA 665 (LINC00665) Gene and the Cell Cycle in Hepatocellular Carcinoma Using The Cancer Genome Atlas, the Gene Expression Omnibus, and Quantitative Real-Time Polymerase Chain Reaction. Med Sci Monit 24:2786-2808. DOI 10.12659/MSM.907389

Cong Z, Diao Y, Xu Y, Li X, Jiang Z, Shao C, Ji S, Shen Y, De W, and Qiang Y. 2019. Long non-coding RNA linc00665 promotes lung adenocarcinoma progression and functions as ceRNA to regulate AKR1B10-ERK signaling by sponging miR-98. Cell Death Dis 10:84. DOI 10.1038/s41419-019-1361-3

Sha QK, Chen L, Xi JZ, and Song H. 2019. Long non-coding RNA LINC00858 promotes cells proliferation, migration and invasion by acting as a ceRNA of miR-22-3p in colorectal cancer. Artif Cells Nanomed Biotechnol 47:1057-1066. DOI $\underline{10.1080 / 21691401.2018 .1544143}$

Gu Z, Hou Z, Zheng L, Wang X, Wu L, and Zhang C. 2018. Long noncoding RNA LINC00858 promotes osteosarcoma through regulating miR-139-CDK14 axis. Biochem Biophys Res Commun 503:1134-1140. DOI 10.1016/j.bbrc.2018.06.131

Zhu SP, Wang JY, Wang XG, and Zhao JP. 2017. Long intergenic non-protein coding RNA 00858 functions as a competing endogenous RNA for miR-422a to facilitate the cell growth in non-small cell lung cancer. Aging-Us 9:475-486. DOI 10.18632/aging.101171

Liu F, Yuan JH, Huang JF, Yang F, Wang TT, Ma JZ, Zhang L, Zhou CC, Wang F, Yu J, Zhou WP, and Sun SH. 2016. Long noncoding RNA FTX inhibits hepatocellular carcinoma proliferation and metastasis by binding MCM2 and miR-374a. Oncogene 35:5422-5434. DOI 10.1038/onc.2016.80

Li X, Zhao Q, Qi J, Wang W, Zhang D, Li Z, and Qin C. 2018. IncRNA Ftx promotes aerobic glycolysis and tumor progression through the PPARgamma pathway in hepatocellular carcinoma. Int J Oncol 53:551-566. DOI 10.3892/ijo.2018.4418

Yang Y, Zhang J, Chen X, Xu X, Cao G, Li H, and Wu T. 2018. LncRNA FTX sponges miR215 and inhibits phosphorylation of vimentin for promoting colorectal cancer progression. Gene Ther 25:321-330. DOI 10.1038/s41434-018-0026-7

He X, Sun F, Guo F, Wang K, Gao Y, Feng Y, Song B, Li W, and Li Y. 2017. Knockdown of Long Noncoding RNA FTX Inhibits Proliferation, Migration, and Invasion in Renal Cell Carcinoma Cells. Oncol Res 25:157-166. DOI 10.3727/096504016X14719078133203

Peer] reviewing PDF | (2019:12:44443:1:0:REVIEW 3 Mar 2020) 
479

480

481

482

483

484

485

486

487

488

489

490

491

492

493

494

495

496

497

498

499

500

501

502

503

504

505

506

507

508

509

Zhang WG, Bi YK, Li JH, Peng F, Li H, Li CG, Wang LZ, Ren FB, Xie C, Wang PW, Liang WW, Wang Z, and Zhu D. 2017. Long noncoding RNA FTX is upregulated in gliomas and promotes proliferation and invasion of glioma cells by negatively regulating miR-342-3p. Laboratory Investigation 97:447-457. DOI 10.1038/labinvest.2016.152

Konecny GE, Wang C, Hamidi H, Winterhoff B, Kalli KR, Dering J, Ginther C, Chen HW, Dowdy S, Cliby W, Gostout B, Podratz KC, Keeney G, Wang HJ, Hartmann LC, Slamon DJ, and Goode EL. 2014. Prognostic and therapeutic relevance of molecular subtypes in high-grade serous ovarian cancer. J Natl Cancer Inst 106. DOI 10.1093/jnci/dju249

Chung SJ, Nagaraju GP, Nagalingam A, Muniraj N, Kuppusamy P, Walker A, Woo J, Gyorffy B, Gabrielson E, Saxena NK, and Sharma D. 2017. ADIPOQ/adiponectin induces cytotoxic autophagy in breast cancer cells through STK11/LKB1-mediated activation of the AMPK-ULK1 axis. Autophagy 13:1386-1403. DOI 10.1080/15548627.2017.1332565

Hanahan D, and Weinberg RA. 2000. The hallmarks of cancer. Cell 100:57-70. DOI 10.1016/S0092-8674(00)81683-9

Delgoffe GM, Pollizzi KN, Waickman AT, Heikamp E, Meyers DJ, Horton MR, Xiao B, Worley PF, and Powell JD. 2011. The kinase mTOR regulates the differentiation of helper T cells through the selective activation of signaling by mTORC1 and mTORC2. Nature Immunology 12:295-U117. DOI 10.1038/ni.2005

Haxhinasto S, Mathis D, and Benoist C. 2008. The AKT-mTOR axis regulates de novo differentiation of CD4+Foxp3+ cells. J Exp Med 205:565-574. DOI 10.1084/jem.20071477

Di Malta C, Siciliano D, Calcagni A, Monfregola J, Punzi S, Pastore N, Eastes AN, Davis O, De Cegli R, Zampelli A, Di Giovannantonio LG, Nusco E, Platt N, Guida A, Ogmundsdottir MH, Lanfrancone L, Perera RM, Zoncu R, Pelicci PG, Settembre C, and Ballabio A. 2017. Transcriptional activation of RagD GTPase controls mTORC1 and promotes cancer growth. Science 356:1188-1192. DOI 10.1126/science.aag2553

Bustelo XR. 2014. Vav family exchange factors: an integrated regulatory and functional view. Small GTPases 5:9. DOI 10.4161/21541248.2014.973757 
510 Figure legend

511 Figure 1 Function enrichment analyses for differently expressed genes.

512 (A) KEGG pathways enrichment for up-regulated genes (red) and down-regulated genes (blue).

513 (B) Biological process terms of GO enrichment for up-regulated genes (blue) and down-

514 regulated genes (orange).

515 Figure 2 ceRNAs interaction network of lncRNA-miRNA-mRNA.

516 It was consisted of 4 DELs (3 upregulated, 1 downregulated) and 8 DEMs (3 upregulated, 5

517 downregulated), 44 DEGs (15 upregulated, 29 downregulated).Square nodes represent lncRNAs;

518 diamond nodes represent miRNAs; circular nodes represent mRNAs. The color presents the fold

519 change (higher expression is red, lower expression is green).

520 Figure 3 ceRNAs interaction network of lncRNA-miRNA-mRNA-KEGG pathway. It was

521 consisted of 3 DELs (2 upregulated, 1 downregulated), 4 DEMs (2 upregulated, 2

522 downregulated), 7 DEGs (4 upregulated, 3 downregulated) and 5 KEGG pathways.

523 Notes: Square nodes represent lncRNAs; diamond nodes represent miRNAs; circular nodes

524 represent mRNAs. The color presents the fold change (higher expression is red, lower expression

525 is green). The blue square nodes represent KEGG pathways.

526 Figure 4 Survival analysis of lncRNAs in ceRNA network.

527 Kaplan-Meier analysis of differentially expressed LINC00665 (A), LINC00858 (B), and FTX

528 (C) in ceRNA network by comparing the higher (red) and lower (green) expressions with overall

529 survival outcomes for patients with HGSOC. P-value set for this analysis is less than 0.05.

530 The bottom part shows the threshold set by $\mathrm{R}$ package. Horizontal axis: the expression level of a

531 certain gene, Vertical axis: standardized Log-Rank statistic. Dotted line: the cutoff value for gene

532 expressions.

533 Figure 5 Correlation of lncRNAs expression with lymphocyte infiltration level in high-grade

534 serous ovarian cancer.

535 (A) LINC00855 has negative correlations with neutrophils and DCs. (B) FTX has negative

536 correlations with infiltrating levels of CD8+ T cells, neutrophils, and macrophages. (C)

537 LINC00665 has significant positive correlations with infiltrating levels of CD4+ T cells, negative

538 correlations with CD8+ T cells, neutrophils, macrophages and DCs.

539

540 Table legend

541 Table 1 Correlation analysis between lncRNAs and relate genes and markers of immune cell

542 after eliminating the influence of tumor purity in HGSOC.

543 Abbreviations: lncRNA, long-non-coding RNA. HGSOC, high-grade serous ovarian cancer.

544 TAM, tumor-associated macrophage. Th (T helper cell), Treg (regulatory $\mathrm{T}$ cell). Cor, $\mathrm{R}$ value of

545 Spearman's correlation.

546 Level of significance: ${ }^{*} \mathrm{p}<0.01 .{ }^{*} \mathrm{p}<0.001 .{ }^{* * *} \mathrm{p}<0.0001$. 
Figure 1

Function enrichment analyses for differently expressed genes.

(A) KEGG pathways enrichment for up-regulated genes (red) and down-regulated genes (blue). (B) Biological process terms of GO enrichment for up-regulated genes (blue) and down-regulated genes (orange).

A

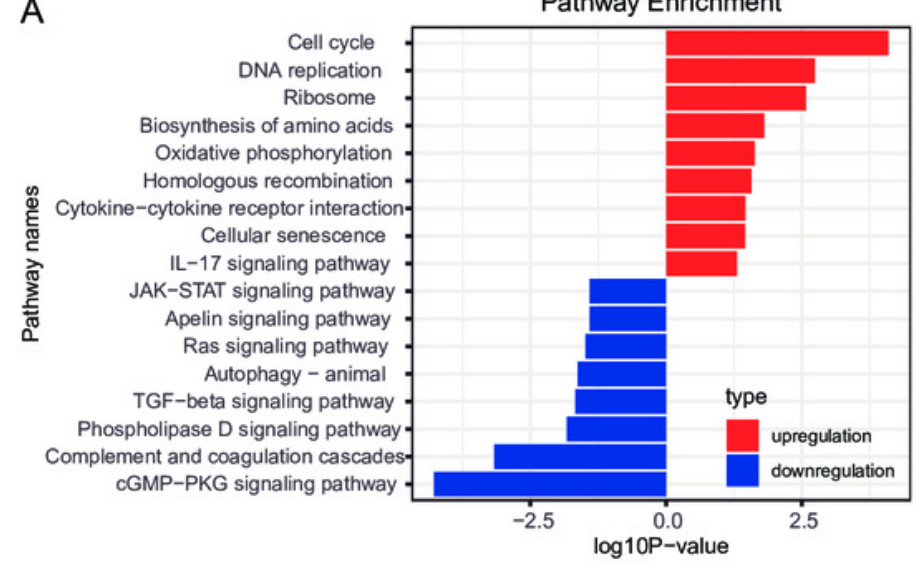

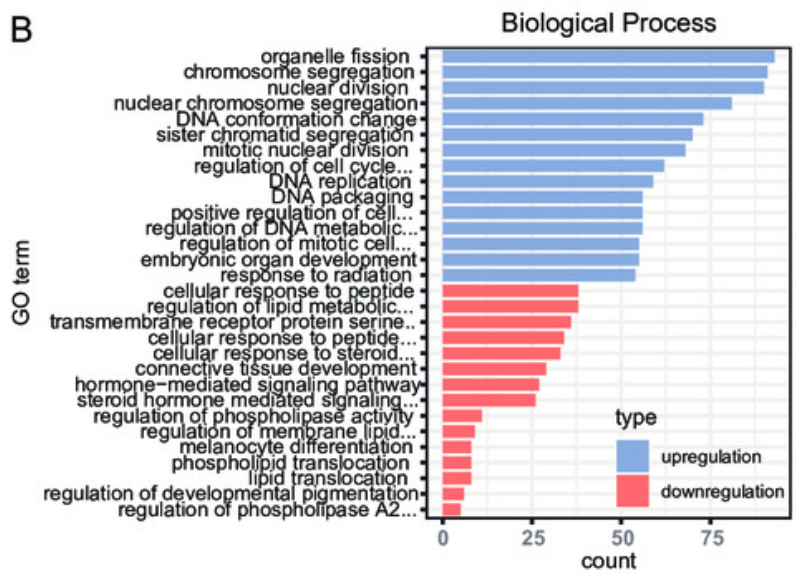




\section{Figure 2}

ceRNAs interaction network of IncRNA-miRNA-mRNA.

It was consisted of 4 DELs (3 upregulated, 1 downregulated) and 8 DEMs (3 upregulated, 5 downregulated), 44 DEGs (15 upregulated, 29 downregulated). Square nodes represent IncRNAs; diamond nodes represent miRNAs; circular nodes represent mRNAs. The color presents the fold change (higher expression is red, lower expression is green). 


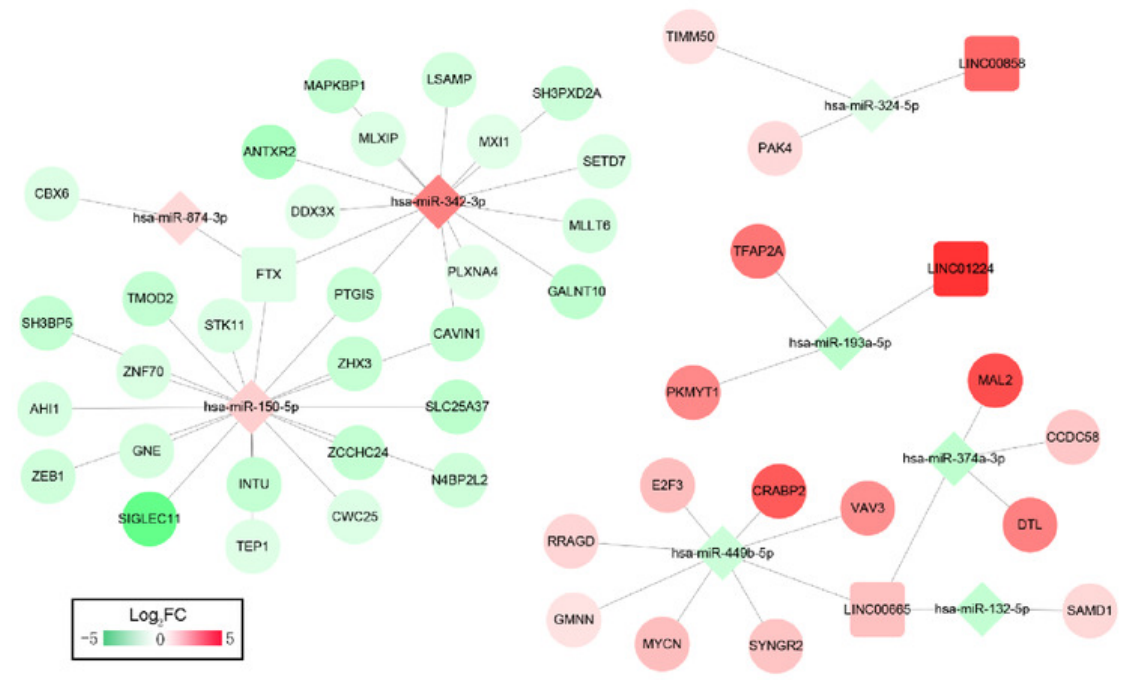




\section{Figure 3}

ceRNAs interaction network of IncRNA-miRNA-mRNA-KEGG pathway.

It was consisted of 3 DELs (2 upregulated, 1 downregulated), 4 DEMs (2 upregulated, 2 downregulated), 7 DEGs (4 upregulated, 3 downregulated) and 5 KEGG pathways. Notes:

Square nodes represent IncRNAs; diamond nodes represent miRNAs; circular nodes represent mRNAs. The color presents the fold change (higher expression is red, lower expression is green). The blue square nodes represent KEGG pathways.

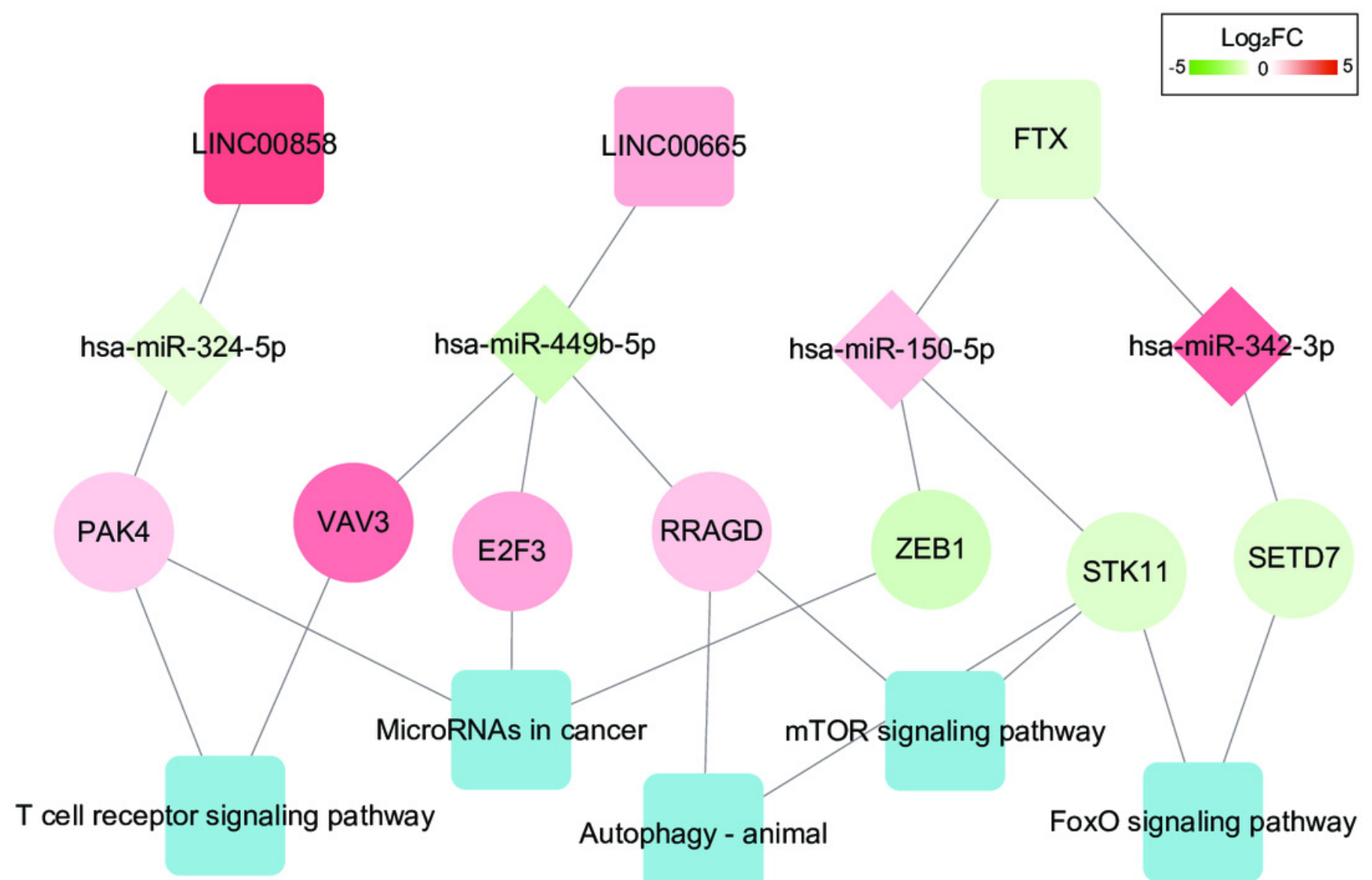




\section{Figure 4}

Survival analysis of IncRNAs in ceRNA network.

Kaplan-Meier analysis of differentially expressed LINC00665 (A), LINC00858 (B), and FTX (C) in ceRNA network by comparing the higher (red) and lower (green) expressions with overall survival outcomes for patients with HGSOC. P-value set for this analysis is less than 0.05 . The bottom part shows the threshold set by R package. Horizontal axis: the expression level of a certain gene, Vertical axis: standardized Log-Rank statistic. Dotted line: the cutoff value for gene expressions.
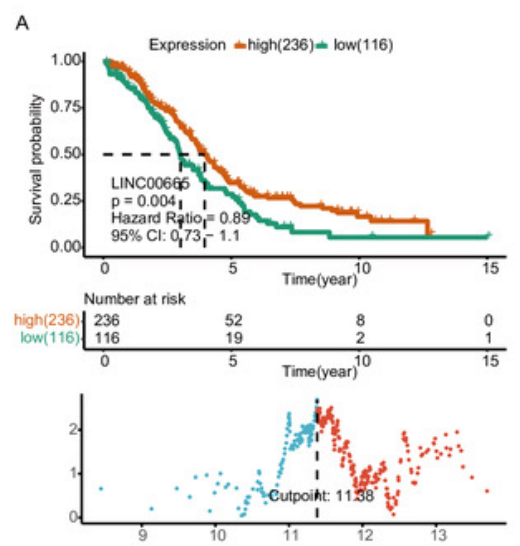
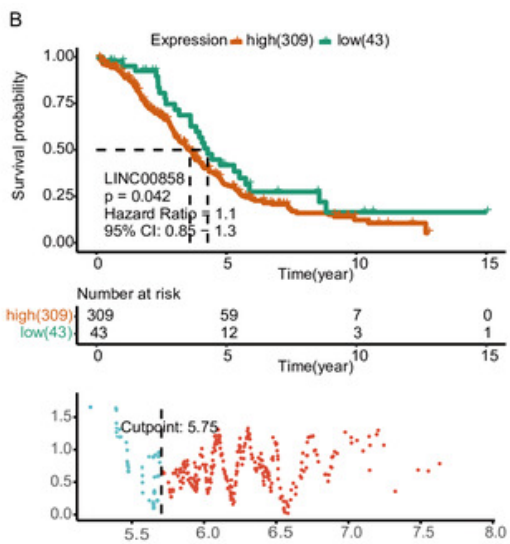
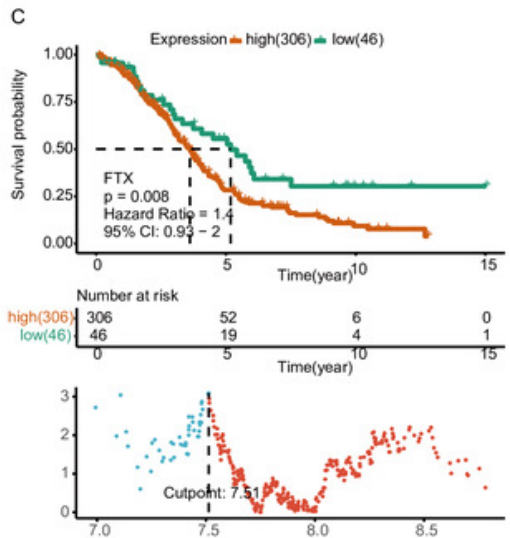
Figure 5

Correlation of IncRNAs expression with lymphocyte infiltration level in high-grade serous ovarian cancer.

(A) LINC00855 has negative correlations with neutrophils and DCs. (B) FTX has negative correlations with infiltrating levels of CD8 + T cells, neutrophils, and macrophages. (C) LINC00665 has significant positive correlations with infiltrating levels of CD4+ T cells, negative correlations with CD8+ T cells, neutrophils, macrophages and DCs.

A

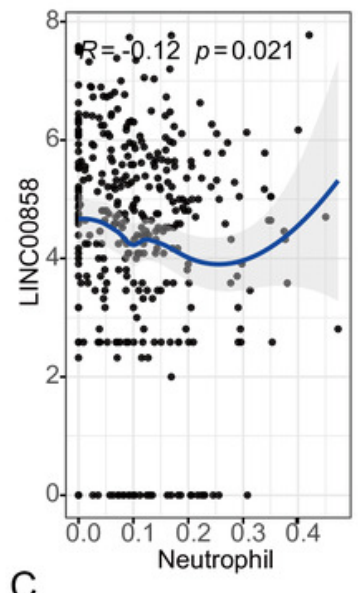

C

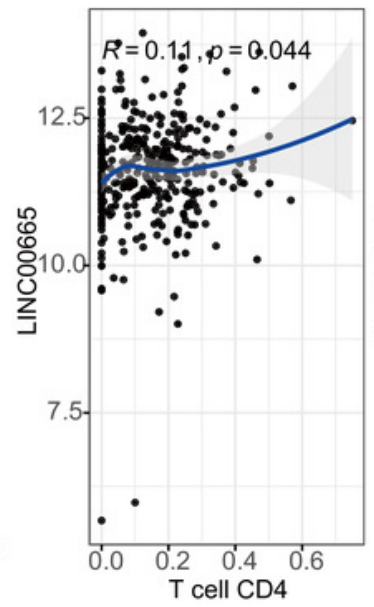

B
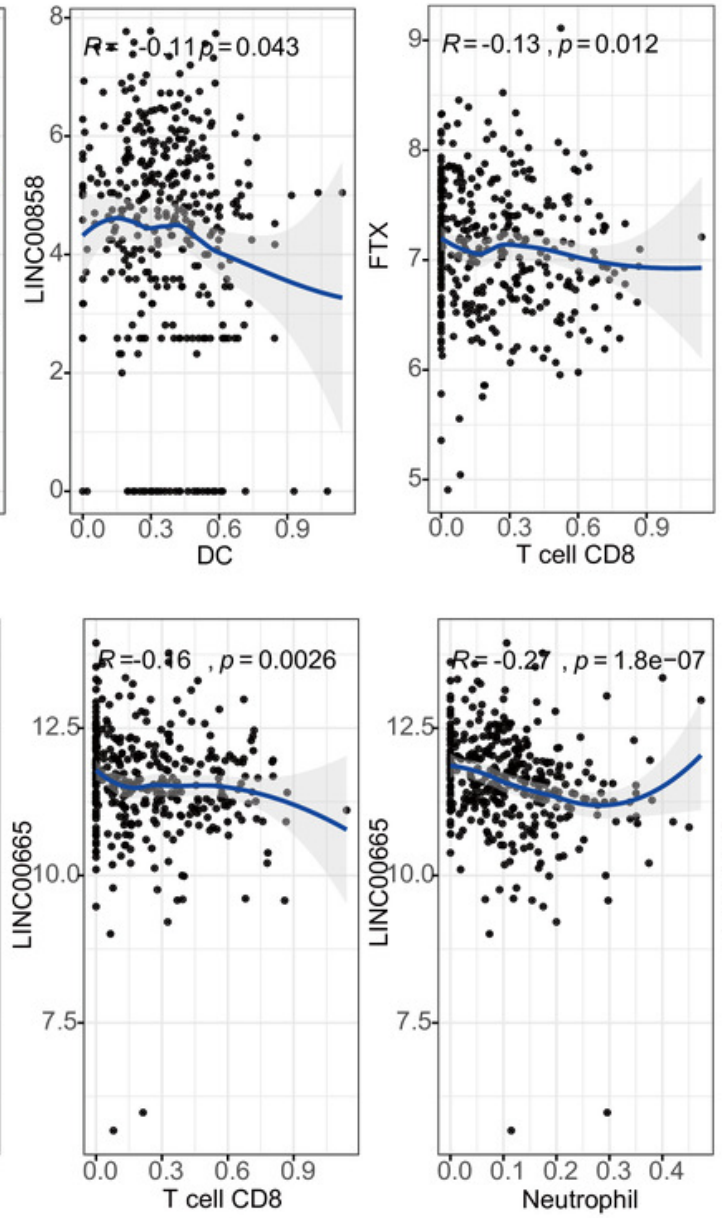
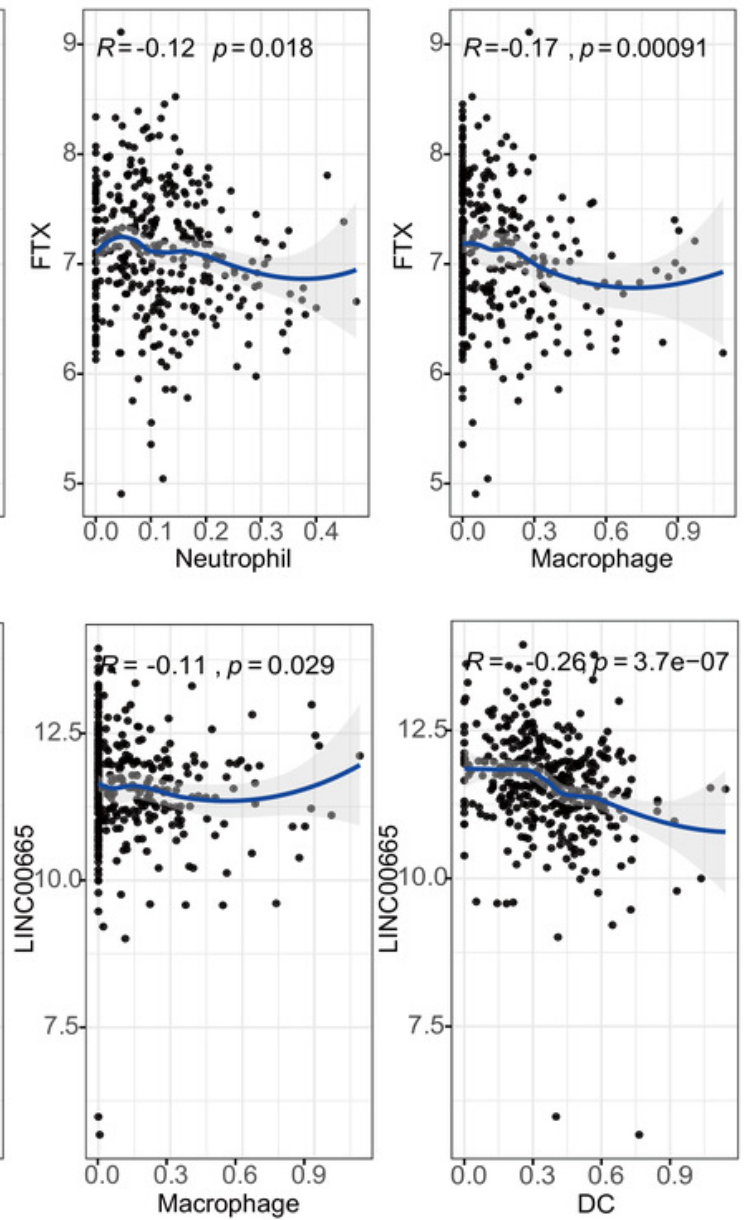


\section{Table $\mathbf{1}$ (on next page)}

Correlation analysis between IncRNAs and relate genes and markers of immune cell after eliminating the influence of tumor purity in HGSOC.

Abbreviations: Abbreviations: IncRNA, long-non-coding RNA. HGSOC, high-grade serous ovarian cancer. TAM, tumor-associated macrophage. Th (T helper cell), Treg (regulatory T cell) . Cor, R value of Spearman's correlation. Level of significance: $* p<0.01$. ${ }^{* *} p<0.001$. $* * * p<0.0001$. 
1 Table 1 Correlation analysis between lncRNAs and relate genes and markers of immune cell after 2 eliminating the influence of tumor purity in HGSOC.

\begin{tabular}{|c|c|c|c|c|c|c|c|}
\hline \multirow[b]{2}{*}{ Description } & \multirow[b]{2}{*}{ Gene markers } & \multicolumn{2}{|c|}{ LINC00858 } & \multicolumn{2}{|c|}{ FTX } & \multicolumn{2}{|c|}{ LINC00665 } \\
\hline & & Cor & $\mathrm{P}$ & Cor & $\mathrm{P}$ & Cor & $\mathrm{P}$ \\
\hline \multirow[t]{2}{*}{ CD8+ T cell } & CD8A & -0.095 & 0.071 & -0.095 & 0.07 & -0.204 & $* * *$ \\
\hline & CD8B & 0.088 & 0.092 & -0.15 & $*$ & -0.078 & 0.14 \\
\hline \multirow[t]{2}{*}{ B cell } & CD19 & -0.016 & 0.762 & -0.018 & 0.738 & 0.07 & 0.186 \\
\hline & CD79A & -0.053 & 0.317 & -0.126 & 0.017 & -0.05 & 0.341 \\
\hline \multirow[t]{3}{*}{ TAM } & CCL2 & -0.166 & * & -0.117 & 0.025 & -0.197 & $* *$ \\
\hline & CD68 & -0.182 & $* *$ & -0.209 & $* * *$ & -0.298 & $* * *$ \\
\hline & IL10 & -0.072 & 0.172 & -0.092 & 0.081 & -0.236 & $* * *$ \\
\hline M1 & NOS2(INOS) & 0.002 & 0.965 & -0.093 & 0.077 & -0.08 & 0.13 \\
\hline \multirow[t]{2}{*}{ Macrophage } & IRF5 & -0.059 & 0.262 & -0.201 & $* *$ & -0.123 & 0.019 \\
\hline & PTGS2(COX2) & -0.044 & 0.4 & -0.078 & 0.137 & -0.068 & 0.198 \\
\hline M2 & CD163 & -0.202 & $* *$ & -0.153 & * & -0.252 & $* * *$ \\
\hline \multirow[t]{2}{*}{ Macrophage } & VSIG4 & -0.136 & * & -0.126 & 0.016 & -0.271 & $* * *$ \\
\hline & MS4A4A & -0.147 & * & -0.187 & $* *$ & -0.288 & $* * *$ \\
\hline \multirow[t]{7}{*}{ Dendritic cell } & HLA-DPB1 & -0.083 & 0.115 & 0 & 0.997 & -0.224 & $* * *$ \\
\hline & HLA-DQB1 & -0.059 & 0.263 & 0.056 & 0.284 & -0.185 & ** \\
\hline & HLA-DRA & -0.102 & 0.052 & -0.02 & 0.71 & -0.213 & $* * *$ \\
\hline & HLA-DPA1 & -0.089 & 0.089 & -0.008 & 0.872 & -0.234 & $* * *$ \\
\hline & CD1C(BDCA-1) & -0.204 & $* * *$ & -0.011 & 0.827 & -0.268 & $* * *$ \\
\hline & NRP1(BDCA-4) & -0.107 & 0.041 & -0.161 & $*$ & -0.111 & 0.034 \\
\hline & ITGAX(CD11c) & -0.165 & $*$ & -0.066 & 0.21 & -0.239 & $* * *$ \\
\hline \multirow[t]{5}{*}{ Th1 } & TBX21(T-bet) & -0.183 & $* *$ & -0.176 & $* *$ & -0.206 & $* * *$ \\
\hline & STAT4 & -0.169 & * & -0.143 & $*$ & -0.238 & $* * *$ \\
\hline & IFNG(IFN- $\gamma)$ & -0.05 & 0.346 & -0.103 & 0.051 & -0.128 & 0.014 \\
\hline & CXCR3 & -0.112 & 0.033 & -0.143 & $*$ & -0.222 & $* * *$ \\
\hline & CCR5 & -0.144 & * & -0.146 & $*$ & -0.279 & $* * *$ \\
\hline \multirow[t]{3}{*}{ Th2 } & GATA3 & -0.128 & 0.015 & -0.061 & 0.244 & -0.212 & $* * *$ \\
\hline & STAT6 & -0.013 & 0.811 & 0.16 & $*$ & -0.097 & 0.064 \\
\hline & CCR4 & -0.133 & 0.011 & -0.114 & 0.03 & -0.245 & $* * *$ \\
\hline \multirow[t]{4}{*}{ Th17 } & STAT3 & -0.067 & 0.203 & -0.227 & $* * *$ & -0.236 & $* * *$ \\
\hline & IL6 & -0.028 & 0.589 & -0.171 & $*$ & -0.142 & * \\
\hline & CCR6 & -0.134 & 0.01 & -0.05 & 0.344 & -0.207 & $* * *$ \\
\hline & $\operatorname{RORA}(\operatorname{ROR} \alpha)$ & 0.041 & 0.44 & 0.129 & 0.014 & -0.144 & $*$ \\
\hline \multirow[t]{4}{*}{ tTreg } & FOXP3 & 0.002 & 0.964 & -0.192 & $* *$ & -0.19 & $* *$ \\
\hline & TGFB1(TGF $\beta)$ & -0.133 & 0.012 & -0.229 & $* * *$ & -0.091 & 0.084 \\
\hline & IL10 & -0.072 & 0.172 & -0.092 & 0.081 & -0.236 & $* * *$ \\
\hline & TBX21(T-bet) & -0.183 & $* *$ & -0.176 & $* *$ & -0.206 & $* * *$ \\
\hline $\mathrm{T}$ cell & PDCD1(PD-1) & -0.034 & 0.522 & -0.193 & $* *$ & -0.173 & $* *$ \\
\hline
\end{tabular}




\begin{tabular}{llllllrr} 
exhaustion & CTLA4 & -0.11 & 0.037 & -0.19 & $* *$ & -0.234 & $* * *$ \\
& LAG3 & -0.096 & 0.066 & -0.135 & $*$ & -0.091 & 0.083 \\
& HAVCR2(TIM-3) & -0.134 & 0.011 & -0.128 & $*$ & -0.297 & $* * *$ \\
& GZMB & -0.085 & 0.107 & -0.108 & 0.04 & -0.169 & $*$ \\
\hline
\end{tabular}

3 Abbreviations: lncRNA, long-non-coding RNA. HGSOC, high-grade serous ovarian cancer. 4 TAM, tumor-associated macrophage. Th (T helper cell), Treg (regulatory $\mathrm{T}$ cell). Cor, $\mathrm{R}$ value of 5 Spearman's correlation.

6 Level of significance: ${ }^{*} p<0.01 .{ }^{* *} p<0.001 .{ }^{* * *} p<0.0001$. 